GUEST EDITORIAL

\title{
Anaesthesia in South Africa
}

'We ought to have saints' days to commemorate the great discoveries which have been made for all mankind, and perhaps for all time - or for whatever time may be left to us. Nature ... is a prodigal of pain. I should like to find a day when we can take a holiday, a day of jubilation when we can fête good Saint Anaesthesia and chaste and pure Saint Antiseptic ... I should be bound to celebrate, among others, Saint Penicillin ... .' (Winston Churchill, speech at Guildhall, London, 10 September $1947^{[1]}$ )

Anaesthesia facilitates performance of surgical and other interventional procedures by quickly, safely and pleasantly producing analgesia, absence of awareness and anxiety, and adequate muscle relaxation. An important aspect of perioperative anaesthetic care is maintenance of physiological homeostasis, including haemodynamic stability, ventilation, oxygenation and temperature. The anaesthetic team may also provide additional services, such as preoperative evaluation, postoperative management in the postanaesthesia care unit or intensive care unit (ICU), and management of both acute and chronic perioperative pain.

Before elective anaesthesia, all patients are evaluated by an anaesthesiologist to assess medical status and preparedness for the planned procedure, implement strategies to reduce risks and create an anaesthetic plan. ${ }^{[2]}$ Fasting guidelines to prevent pulmonary aspiration of gastric contents are applied in all patients undergoing elective surgery, including procedures performed under general anaesthesia (GA), regional anaesthesia, and monitored anaesthesia care (MAC). Prediction of the degree of difficulty of airway management and ventilation is an important part of the preanaesthetic evaluation.

The American Society of Anesthesiologists (ASA)'s physical status classification system is relatively simple. It has proven effective in stratifying overall perioperative risk of morbidity and mortality for patient-specific risk factors (Table 1). ${ }^{[3]}$ Patients are classified according to the degree to which underlying medical problems produce functional limitations, and a higher ASA physical status is associated with increased risk of complications, unexpected hospital admission after surgery, postoperative admission to the ICU, longer duration of hospital stay, and higher costs and mortality due to patient- and surgery-specific factors. ${ }^{[3]}$ Risk assessment tools that combine patient and surgical risk factors have been developed. In older adults, frailty predicts postoperative mortality, morbidity,

Table 1. American Society of Anesthesiologists physical status classification system

\begin{tabular}{ll}
\hline $\begin{array}{l}\text { ASA physical status } \\
\text { classification }\end{array}$ & Definition \\
\hline ASA I & A normal healthy patient \\
ASA II & A patient with mild systemic disease \\
ASA III & $\begin{array}{l}\text { A patient with severe systemic disease } \\
\text { A patient with severe systemic disease that } \\
\text { ASA IV }\end{array}$ \\
is a constant threat to life \\
ASA V & $\begin{array}{l}\text { A moribund patient who is not expected to } \\
\text { survive without the operation }\end{array}$ \\
ASA VI & $\begin{array}{l}\text { A declared brain-dead patient whose organs } \\
\text { are being removed for donor purposes }\end{array}$ \\
ASA = American Society of Anesthesiologists.
\end{tabular}

discharge disposition and functional decline. ${ }^{[4]}$ Anaesthesia-related mortality rates have decreased to 1 death per 200000 - 300000 anaesthetics in the last few decades, ${ }^{[5]}$ primarily as a consequence of improvements in the understanding of physiology, anaesthesia equipment and monitoring, anaesthetic agents and techniques, and a greater focus on anaesthesia safety.

Factors affecting the selection of appropriate anaesthetic techniques for an individual patient include surgical requirements for performance of the procedure, anticipated duration of surgery, patient comorbidities and preferences, plans for providing postoperative analgesia, and experience and preferences of the anaesthesia care provider. As a general rule, there are no clear-cut indications for one type of anaesthesia over another when either would be appropriate. ${ }^{[6]}$ For major or prolonged procedures, GA with airway management using an endotracheal tube or supraglottic airway device is usually the most appropriate primary technique, particularly if a deep level of sedation is required and/or if airway access is limited. GA may also be selected for minor procedures, particularly if it is the patient's preference. Depending on the location of the procedure, neuraxial anaesthesia (e.g. peripheral nerve block, intravenous regional anaesthesia), or local anaesthetic infiltration by the surgeon, may be selected. These techniques are often supplemented with lighter levels of sedation to maintain airway reflexes (i.e. sedation with MAC).

There are benefits and risks to any type of anaesthetic. When assessing whether avoidance of GA is appropriate, considerations include the patient's ability to lie motionless in the position required for the procedure, and whether the patient can co-operate, communicate and is willing to undergo the procedure, given the possibility of awareness with recall. In many cases, a combined technique (i.e. GA plus epidural or peripheral nerve block for supplemental analgesia) provides both optimal intraoperative conditions and excellent multimodal management of postoperative pain.

Standard monitoring during anaesthesia includes basic physiological measures such as pulse oximetry, temperature, electrocardiography and non-invasive blood pressure devices. ${ }^{[7]}$ Furthermore, for ventilated patients, measurement of end-tidal carbon dioxide and inspired oxygen concentration, as well as use of low oxygen concentration and ventilator disconnect alarms, is helpful. Quantitative monitoring of the volume of expired gas is strongly encouraged. In patients undergoing GA, end-tidal inhalation anaesthetic concentrations are measured to aid in monitoring anaesthetic depth and preventing awareness. Neuromonitoring with electroencephalography provides supplemental information. Invasive haemodynamic monitoring requires insertion of an intra-arterial, central venous or pulmonary artery catheter, or a transoesophageal echocardiography probe. Maintenance of perioperative haemodynamic stability, including fluid administration to maintain optimal intravascular volume status and use of vasoactive drugs when necessary, is based on assessment of standard and/or invasive haemodynamic monitoring parameters.

Ideally, after having received anaesthesia, all patients should be monitored in a post-anaesthesia care unit that provides standardised assessment of recovery, including discharge criteria, resulting in reduced postoperative adverse events and streamlined postoperative care. ${ }^{[8]}$ After MAC with minimal sedation, patients 
who have completely recovered may proceed directly to the predischarge unit for more rapid discharge. Critically ill patients and those who are intubated are admitted directly to the ICU.

Commenting on the state of surgery and anaesthesia in South Africa (SA), Patel et al. ${ }^{[9]}$ remarked, 'Considering the challenges identified, developing a national road map for surgery and anaesthesia is a colossal task. Objective assessment of population needs is vital to developing any strategies aimed at addressing deficiencies in the current profile of service delivery. This assessment demands accurate and regular data collection and reporting. In spite of the existing demands on service delivery providers, an ongoing assessment of needs and the effectiveness of policy interventions provides an opportunity for innovation and to develop the relationship between tertiary, regional, peripheral and primary levels of care'

In an editorial in 2012, Diedericks ${ }^{[10]}$ wrote, 'In SA the majority of anaesthetics are provided by non-specialists. In most cases this involves anaesthesia for small, brief procedures. Many of these cases are anaesthesia for caesarean section. Unfortunately the reports on maternal death indicate that SA has a relatively high rate of deaths associated with anaesthesia for caesarean section. This may be an indicator of all anaesthesia care. Many factors contribute to this situation, but the loss of experienced practitioners in rural areas, inadequate training, and high turnover of personnel are factors that are important.' In 2018, sadly, the state of anaesthesia in SA has not changed significantly from the description given above.

In this issue of CME, several topics are reviewed that hold great relevance for non-specialists and specialists alike; it is hoped that these will improve the practice of anaesthesia in SA. First, advances in paediatric anaesthesia are considered. Availability of dedicated facilities with appropriate equipment, monitoring and specialised personnel trained in paediatric anaesthesia and resuscitation have contributed to the reduction in perioperative risk for paediatric patients. Bester et al. ${ }^{[11]}$ provide guidance on the selection of suitable facilities for perioperative care, risk stratification and patient selection, safe selection of medication and standardisation of its use, and implementation of specific anaesthetic techniques that can minimise risk in the paediatric surgical population.

Second, the management of spinal hypotension and associated complications following caesarean section is reviewed. ${ }^{[12]}$ Spinal hypotension may be predicted by simple parameters such as age $>25$ years, preoperative heart rate $>90 \mathrm{bpm}$ and preoperative mean arterial pressure $<90 \mathrm{mmHg}$. Heart rate variability and point-ofcare echocardiography predict hypotension with greater accuracy. Spinal anaesthesia is absolutely contraindicated if the patient is hypovolaemic. Left lateral tilt is advised. The dose of spinal bupivacaine should not be reduced in obese patients. Crystalloid co-loading is an adequate fluid strategy in most cases, but of limited efficacy in the prevention of hypotension. Early intervention with phenylephrine is the first-line approach for hypotension if heart rate is preserved under spinal anaesthesia. The vigilant use of phenylephrine boluses, targeting maternal heart rate as a surrogate for cardiac output, is effective.

Third, the management of myocardial injury after non-cardiac surgery (MINS) is reviewed. ${ }^{[13]}$ MINS is defined as an elevated postoperative cardiac troponin level resulting from myocardial ischaemia, without evidence of a non-ischaemic cause. The perioperative context contributes to the pathophysiology of relative myocardial hypoperfusion and ischaemia, which differentiates MINS from myocardial infarction in non-surgical patients. More than $80 \%$ of MINS patients are asymptomatic for myocardial ischaemia, and therefore would not fulfil the universal definition of myocardial infarction, despite a similar prognosis. Accurate diagnosis of MINS therefore relies on routine postoperative cardiac troponin surveillance for 48 - 72 hours, which is cost-effective, even in SA. One in 10 patients with MINS dies within 30 days of surgery, and 1 in 5 sustains major cardiovascular complications. Simple treatment strategies could improve short- and long-term mortality, which include cardioprotective therapy intensification, and ensuring therapy with aspirin and statins.

It is my hope that the series of articles included in the CME issues on perioperative medicine and anaesthesia will improve knowledge, enhance practice, and translate into improved care of SA patients.

Funding. This manuscript is not funded. Prof. N Ntusi gratefully acknowledges support from the National Research Foundation and the Medical Research Council of South Africa, as well as the Harry Crossley Foundation

Conflicts of interest. None.

\section{Ntobeko Ntusi}

Division of Cardiology, Department of Medicine, Faculty of Health Sciences, University of Cape Town and Groote Schuur Hospital; Cape Universities Body Imaging Centre, Faculty of Health Sciences, University of Cape Town; and Hatter Institute of Cardiovascular Research in Africa, Department of Medicine, Faculty of Health Sciences, University of Cape Town, South Africa

ntobeko.ntusi@uct.ac.za

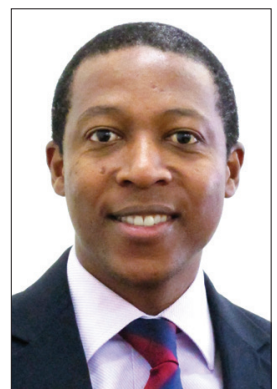

Gaither CC, Cavazos-Gaither AE, Gaither's Dictionary of Scientific Quotations. 2nd ed. New York: Springer, 2012:1382.

2. Committee on Standards and Practice Parameters, Apfelbaum JL, Connis RT, Nickinovich DG; American Society of Anesthesiologists Task Force on Preanesthesia Evaluation, Pasternak LR, Arens JF, Caplan RA, et al. Practice advisory for preanesthesia evaluation: An updated report by the American Society of Anesthesiologists Task Force on preanesthesia evaluation. Anesthesiology 2012;116(3):522538. https://doi.org/10.1097/ALN.0b013e31823c1067

3. Cohen MM, Duncan PG, Tate RB. Does anesthesia contribute to operative mortality? JAMA 1988;260(19):2859-2863. https://doi.org/10.1001/jama.1988.03410190107032

Kim SW, Han HS, Jung HW, et al. Multidimensional frailty score for the prediction of postoperative mortality risk. JAMA Surg 2014;149(7):633-640. https://doi.org/10.1001/jamasurg.2014.241

5. Botney R. Improving patient safety in anesthesia: A success story? Int J Radiat Oncol Biol Phys 2008;71(1 Suppl):S182-S186. https://doi.org/10.1016/ijijrobp.2007.05.095

6. Urwin SC, Parker MJ, Griffiths R. General versus regional anaesthesia for hip fracture surgery: A meta-analysis of randomized trials. Br J Anaesth 2000;84(4):450-455. https://doi.org/10.1093/ A meta-analysis of rando

7. Committee of Origin: Standards and Practice Parameters. Standards for basic anesthetic monitoring. 2016. https://www.asahq.org/ /media/Sites/ASAHQ/Files/Public/Resources/standards-guidelines/standardsfor-basic-anesthetic-monitoring.pdf (accessed 6 May 2018).

8. Apfelbaum JL, Silverstein JH, Chung FF, et al.; American Society of Anesthesiologists Task Force on Postanesthetic Care. Practice guidelines for postanesthetic care: An updated report by the American Society of Anesthesiologists Task Force on postanesthetic care. Anesthesiology 2013;118(2):291-307. https://doi.org/10.1097/ALN.0b013e31827773e9

9. Patel N, Peffer M, Leusink A, Singh N, Smith M. Surgery and anaesthesia in the South African context: Looking forward. S Afr Med J 2016;106(2):135-136. https://doi.org/10.7196/SAMJ.2016.v106i2.10529 10. Diedericks J. Anaesthetics. CME 2012;30(6):192.

11. Bester K, Meyer H, Crowther M, Gray R. Anaesthesia for paediatric patients: Minimising the risk. S Afr Med J 2018;108(6):457-459. https://doi.org/10.7196/SAMJ.2018.v108i6.13351

2. Gibbs MW, van Dyk D, Dyer RA. Managing spinal hypotension during caesarean section: An update. S Afr Med J 2018;108(6):460-463. https://doi.org/10.7196/SAMJ.2018.v108i6.13373

3. Coetzee E, Biccard BM. Myocardial injury after non-cardiac surgery: Time to shed the ignorance, S Afr Med J 2018;108(6):464-467. https://doi.org/10.7196/SAMJ.2018.v108i6.13346 